\title{
Society for Pediatric Research Presidential Address 2011: improving child health outcomes-from 65 roses to high reliability
}

\author{
Maria T. Britto'
}

lisions

n 1965, the life span of people with cystic fibrosis (CF) was less than $10 \mathrm{y}$. Mary Weiss was the mother of three children with CF-Richard, Arthur, and Anthony-and she was doing what parents, especially those whose children have a severe chronic illness, do: she was advocating for her kids. In her case, she was a volunteer, placing phone calls to raise money for $\mathrm{CF}$ research, which her son Richard heard not as "cystic fibrosis," but rather as "65 Roses", and a moniker and symbol for the fight against CF was born (1).

Improving outcomes for children, especially those with a chronic disease like $\mathrm{CF}$, requires a network of clinicians, researchers, educators, and advocates. But at the center of that network are the families and the patients. Parents and patients can be some of our best and most persistent accelerators of improvement in care. Polly Arango was one such parent. Her son Nicholas had severe developmental disabilities and, in the course of working to provide for his educational and health care needs, she founded Family Voices, in 1992 (2). This organization, which has grown to be national in scope, works with parents to increase their ability to care for and advocate for their children and with health care organizations and policy makers to create systems and policies that enhance family-centered care for all children. I came to know Polly through her work with the National Initiative for Children's Health Care Quality and later with the Institute for Healthcare Improvement. Polly died this past June in a car crash, but she exemplified the passion, energy, and expertise that parents can bring to our effort to improve health care.

I was 4 y old when Mary Weiss was making phone calls to support CF research, but I was a second-year internal medicine and pediatrics resident at the University of North Carolina (UNC), deep in the details of tobramycin peaks and troughs in September 1989, when Science published the report of the cloning of the CF Transmembrane Receptor (CFTR) gene (3). At that time, at UNC, we admitted more children and adults with CF than with asthma, and we, like others, were hopeful that a major change in therapy and outcomes would soon follow.

In more recent years, the sequence and interdependency among the stages of the research process have been formalized in conceptual frameworks and roadmaps developed for moving from basic discoveries in the laboratory to routine implementation in practice that actually leads to the improved outcomes that matter to clinicians and families. In an article in JAMA in 2008, Dougherty and Conway described a "3Ts" roadmap for transforming US health care (4). Their framework shows that not only do we need what we usually consider typical or T1 research to bring basic discoveries to the clinic, and also a second phase of translation (T2) (using methods from health services and comparative effectiveness research to determine who will benefit from new approaches), we also need a third level of translation to determine how to deliver high-quality care reliably in all care settings (T3). This stage of translation requires new methods that have been less applied in health care, such as quality improvement and implementation research.

While finding an effective therapy for CF based on genetic knowledge has proven more challenging than anticipated, this discovery and its associated techniques formed some of the early foundation that eventually led to the work that we heard described today by Drs Hirschorn and Villain, our E. Mead Johnson Awardees.

In the meantime, other therapeutic and care advances in CF led to significant improvements in quality of life and increases in life expectancy, as typified by the healthy-appearing now 24-y-old Danny Bessette, who accompanied a 20-year retrospective on the discovery of the CF gene in 2009 (5). Nonetheless, at about the same time as CFTR was being sequenced, the CF Foundation began analyzing its national registry data in new ways that uncovered huge variations in care and outcomes across CF Centers in the United States. In 2002, when the CF Foundation compared the proportion of patients in nutritional failure across its accredited CF Care Centers, it found wide variation. At the best-performing center, only $18 \%$ of patients were in nutritional failure as compared to nearly $60 \%$ at the center with the worst outcome. While some of this variation was due to differences in patient profiles, the CF registry data also showed that there was much variation in care processes among the centers, and that few centers delivered key elements of care to even the majority of their patients. 


\section{Special Article | Brito}

This variation and poor performance in care processes is not limited to CF. A report by Mangione-Smith and colleagues, published in the New England Journal of Medicine in 2007, titled "The Quality of Ambulatory Care Delivered to Children in the United States" (6), described shortfalls in the quality of care delivered to children in the United States. Overall, they found that children received only $46.5 \%$ of recommended ambulatory care. The system performed best for acute care, reaching $68 \%$ overall in that category, topping out at $97 \%$ for care of upper respiratory infections. The worst performance was for adolescent preventive services, where only $35 \%$ of patients received the required services.

Returning to $\mathrm{CF}$, by the time the data on the variation in quality of care and outcomes were made available by the CF Foundation, I was a junior researcher at Cincinnati Children's Hospital studying quality of care for children with chronic illness, and with CF in particular. The data on nutritional failure for Cincinnati showed that we were only somewhat above average. We had just begun our first major quality improvement efforts, and we had a willing partner in Bob Wilmont, who was the CF Center director at the time. We were part of a national effort to learn how to improve care and outcomes for whole systems, and transparency about results was part of that effort. We brought parents to the table to help us in our improvement efforts, and we shared these data and other data with them (7).

Three of our most engaged parents were Jim Lang, Tracy Blackwelder, and Honor Page. At the time, we were newbies in the area of improving the quality of care, and the parents who became an integral part of our team were truly accelerators of the work. They looked at the data with us; they asked hard questions; they realized we were committed to change; and they stepped up to help make change happen. When we got bogged down with issues such as lack of strong data on which to base certain decisions, or when we got wrapped up in traditional roles, they were the ones who reminded us that the clock was ticking for their kids and that we had better not be wasting time.

So, how did we begin to change CF care? At about the same time that the CF Foundation was looking at its data in new ways, the Institute of Medicine produced its report on health care quality titled "Crossing the Quality Chasm" (8), with which I am sure most of you are familiar. It said the current health care system cannot do the job, and trying harder will not work. Changing the system could work, and it laid out a roadmap for doing so. The science for understanding and changing complex adaptive systems like health care comes from disciplines far beyond usual biomedicine such as cognitive psychology, sociology, and engineering. Because so many health-care processes were performing so poorly, we needed to figure out how to increase the reliability of those processes so that we could consistently deliver the care that the science tells us will help patients.

Tom Nolan, a statistician; Roger Reser, a physician and safety researcher; and others developed a framework to translate findings from these disciplines and from other industries and make them applicable to health care (9). Basically, they said we cannot depend on human beings to deliver even the most critical elements of care-such as site preparation for a surgical procedure $-100 \%$ of the time, especially for complex care. Therefore, we need to put in place other systems to ensure that the right thing happens consistently.

They defined reliability as "the inverse of a systems failure rate." For example, if the system fails 1 time in 10, then its level of performance is $10^{-1}$. Using principles from the literature, they suggested three major approaches to improving reliability. The first of these is the prevention of failures. Examples include the use of guidelines and clinical algorithms to drive care. Memory aids, such as color coding of different streams of care, are another method of preventing failures. The second design principle is to identify failures early on and to mitigate them, either before they affect the patient at all, or at least in time to reduce the impact on the patient. An example of this would be to actively check orders to determine whether they meet key guideline recommendations and, if not, to actively pursue the remediation of those orders. Another example of improving reliability involves independent double-checking of medications and other forms of redundancy of procedures. The third class of intervention to improve reliability includes complete redesigning of the process based on the causes of critical failures. A technique that is used to do this starts with failure-modes analysis. All the ways in which the system can fail are identified, and then each of those failure modes is systematically addressed when redesigning the system, so that the process is highly unlikely to fail. High-reliability concepts can be applied in high-risk environments anywhere in the healthcare system where there are processes with multiple steps, and where failure is likely to lead to harm or lack of effectiveness. Operating rooms, intensive care units, and chemotherapy centers are such high-risk environments.

One characteristic of pediatricians and pediatric researchers is that we are willing to work together. In the context of chronic disease it is required that we do this, because rarely will one center have enough patients to allow any definitive conclusions to be drawn from the available data. The Children's Oncology Group is an obvious example of the power of these collaborations. When it comes to systems improvement, by working together we can learn from variation in outcomes, but also from how the best-performing organizations provide care and how we can spread these practices to other organizations. Sixty pediatric intensive care units across the United States worked together to systematically reduce catheter-associated bloodstream infections. One of the main interventions involved using the reliability principles described earlier to implement a group or "bundle" of care practices related to the insertion and maintenance of the catheter. The results from the first of 29 pediatric intensive care units which were engaged in this work were recently published. These teams found that the use of the reliability principles to implement the care bundles at high rates (later also using chlorahexadine scrubs or sponges) dramatically reduced the rate of bloodstream infection. The rate decreased $56 \%$, from 5.2 to 2.3 infections per 1,000 line-days (10). This work has been extended into 60 pediatric intensive care units, and the investigators estimate that their work has saved 62 lives per year and \$18 million in health-care costs per year. 
In order to improve care and outcomes for children with chronic diseases like CF, additional steps are needed. There is a strong evidence base to provide guidance to those who wish to improve health-care systems. This evidence comes from a Cochrane review and other studies that were completed in 2002 $(11,12)$. There were 40 studies, $85 \%$ of which were randomized controlled trials, mostly in the area of adult primary care. There were four major categories of interventions that were investigated. These included decision support, delivery system design, changes to information systems, and self-management support. Self-management appeared to be very important for improving outcomes in chronic disease. Of the 20 studies that included self-management support, 19 showed positive effects. It also appears likely that multiple interventions in multiple categories of delivery system design are necessary to get the best outcomes. All five of the studies that included all four categories of interventions had positive effects.

Clinician teams caring for children and adolescents with inflammatory bowel disease in a network called "Improve Care Now" have worked together to improve care and outcomes for these patients, using the model just described. For example, by redesigning the delivery system and providing point-of-clinical-care decision support systems, this collaborative increased the number of encounters where all components of the inflammatory bowel disease classification bundle were completed from 22\% at baseline in 2007 to $85 \%$ by 2009 . Using this and a number of other improvements to the system, including feedback to teams, self-management support, and pre-visit planning, the remission rate for this population improved from approximately $45 \%$ at the beginning of the collaborative intervention to almost $75 \%(13,14)$.

These examples of intensive care physicians and gastroenterologists working together in networks to improve quality and outcomes show the power of working across organizations. While clinicians network, and certainly researchers network, patients network as well. The Internet has facilitated direct networking among patients both through general-use forums such as Facebook and disease-related organizations such as PatientsLikeMe (15). Right now, we at the Pediatric Societies are networking with one another, reconnecting with old colleagues, and meeting new ones.

We tend to think of social networks as something newly created and found in a virtual dimension on the Internet. However, social networks theory has been developed in the field of sociology since at least the early 1970s. Mark Granovetter at Johns Hopkins published a seminal work called "The Strength of Weak Ties," in the American Journal of Sociology in 1973 (16). His work and others have demonstrated that, while strong ties (those among tightly interconnected individuals such as one's clinical unit or one's research lab team) are very important, these strong ties are in some ways less influential than weak ties. Weak ties are the bonds between groups, each of which has strong ties among the individuals within it. These weak ties are the ones you may have with a researcher who is at another organization and with whom you do not work directly, or the ties you may have with those with whom you shared residency or fellowship. When there are no ties among a given set of groups, there is said to be a "structural hole". Individuals who bridge structural holes and bring together groups to create new weak ties between them can be highly influential; they are sometimes known as "connectors." This idea of connectors and their power was popularized by the author Malcolm Gladwell in his book The Tipping Point in the year 2000 (17). Gladwell suggests that connectors share some common characteristics. First of all they know a large number of people. They tend to have social circles that are four or five times larger than that of the average person. In addition, they often tend to occupy many different worlds and subcultures. For example, within health care, a connector might be involved in a specific clinical specialty area (i.e., liver transplant); he might also be involved in quality improvement for a hospital system and in clinical trials. In addition, he may engage with surgeons and with persons involved in the improvement of chronic disease care systems besides being active in community organizations and national organizations unrelated to his professional work. Such individuals are ideally suited for bridging gaps between groups by spotting opportunities to bring them together, and for creating new ideas and new interventions.

How does all of this relate to the topic with which we started today-improving outcomes for children with CF? Through the use of quality improvement approaches such as reliability interventions and the chronic care model, and by improving social networking among patients, care providers, and the CF Foundation, the CF community has achieved a dramatic improvement in outcomes, as shown by data from Gerry O'Connor of the Cystic Fibrosis Foundation. In 1999, as I mentioned previously, 35\% of CF Centers were below the median value as regards both lung function and body mass index percentile. However, by 2006, only $5.8 \%$ of the Centers were below the 1999 median values for these parameters, and fully $61 \%$ of all the Centers were in the upper half of the respective percentiles for lung function (FEV1) and BMI. This translates into a highly significant improvement in survival for patients with CF in the United States. Between 2002 and 2006, during which period there were no substantive new therapies available for $\mathrm{CF}$, there was nevertheless active quality-improvement work under way, and the predicted survival improved from 28.6 to $36.9 \mathrm{y}$ across the country. This translates into 741 additional lives saved. Therefore, if we were to consider the degree of improvement and lives saved in CF, and extend that to all of the chronic conditions that we come across in children, we would get an idea of the number of children's lives that could be saved. Furthermore, if we can create reliable systems of health care that can operate in such a way as to integrate new evidence into care quite quickly, we can transform the paradigm so that new therapies are quickly integrated into routine care and all patients will benefit more quickly.

So, what can we do today while we are here at the Pediatric Academic Societies? First, I urge you to build some new nodes in your own social network. Go and attend a talk or workshop that is outside your area of expertise. Follow up with at least 


\section{Special Article | Brito}

two of the new people that you met while you have been here. Reconnect with an old colleague. And, perhaps most important, involve patients and families in your work. It will connect you to a whole world of inspired and motivated people who can help change the way we think and thereby help to improve the quality and delivery of care in chronic pediatric diseases.

In closing, I would like to say thank you to all of my weak and strong ties. First, my gratitude to the Society for Pediatric Research (SPR) Council and all of the members of the SPR committees and task forces. The strength of the SPR lies in the network of all of its members, but these individuals work particularly hard to represent you and to help drive improved quality, not only for the Pediatric Academic Societies meeting but for our initiatives throughout the year. I also thank all of the staff of the SPR in the Woodlands, particularly Debbie Anagnostelis who is the American Pediatric Society and SPR executive director. Debbie was unable to be here today, but she is very much in our thoughts. I would also like to thank my colleagues from around the country who are both my strong and weak ties; my mentors and colleagues in Cincinnati, including Tom Boat, who was my first clinical attending when I was an intern at UNC and with whom I have been privileged to work throughout much of my career; my current chair, Arnie Strauss; my division directors, Uma Kotagal in the James Anderson Center for Health Systems Excellence and Frank Biro in the Division of Adolescent Medicine; all of my colleagues at Cincinnati Children's Hospital, and the countless others with whom I work every day to improve children's health. Finally, I would like to thank my parents, Kay and Nelson Britto, who are here today. They provide my strongest ties and are perhaps my strongest evidence that parents always matter. Thank you.

\section{REFERENCES}

1. Cystic Fibrosis Foundation. About 65 Roses, 2006. (http://www.cff.org/ aboutCFFoundation/About65Roses/.) Accessed 14 June 2011.
2. FamilyVoices. Keeping Families at the Center of Children's Health Care, 2011. (http://www.familyvoices.org/.) Accessed 14 June 2011.

3. Koshland DE Jr. The cystic fibrosis gene story. Science 1989;245:1029.

4. Dougherty D, Conway PH. The "3T's" road map to transform US health care: the "how" of high-quality care. JAMA 2008;299:2319-21.

5. Pearson H. Human genetics: One gene, twenty years. Nature 2009;460:164-9.

6. Mangione-Smith R, DeCristofaro AH, Setodji CM,et al. The quality of ambulatory care delivered to children in the United States. N Engl J Med 2007;357:1515-23.

7. Britto MT, Anderson JM, Kent WM,et al. Cincinnati Children's Hospital Medical Center: transforming care for children and families. Jt Comm J Qual Patient Saf 2006;32(10):541-8.

8. Leavitt M. Medscape's response to the Institute of Medicine Report: Crossing the quality chasm: a new health system for the $21^{\text {st }}$ century. MedGenMed 2001;3:2.

9. Nolan R, Resar R, Haraden C, Griffin FA. Improving the Reliability of Health Care. IHI Innovation Series white paper. Boston: Institute for Healthcare Improvement, 2004. (http://www.ihi.org/knowledge/Pages/ IHIWhitePapers/ImprovingtheReliabilityofHealthCare.aspx.)

10. Miller MR, Niedner MF, Huskins WC, et al.; National Association of Children's Hospitals and Related Institutions Pediatric Intensive Care Unit Central Line-Associated Bloodstream Infection Quality Transformation Teams. Reducing PICU central line-associated bloodstream infections: 3-year results. Pediatrics 2011;128:e1077-83.

11. Bodenheimer T, Wagner EH, Grumbach K. Improving primary care for patients with chronic illness: the chronic care model, Part 2. JAMA 2002;288(15):1909-14.

12. Bodenheimer $\mathrm{T}$, Wagner $\mathrm{EH}$, Grumbach $\mathrm{K}$. Improving primary care for patients with chronic illness. JAMA 2002;288(14):1775-9.

13. Linam WM, Margolis PA, Atherton H, Connelly BL. Quality-improvement initiative sustains improvement in pediatric health care worker hand hygiene. Pediatrics 2011;128:e689.

14. Crandall WV, Boyle BM, Colletti RB, Margolis PA, Kappelman MD. Development of process and outcome measures for improvement: lessons learned in a quality improvement collaborative for pediatric inflammatory bowel disease. Inflamm Bowel Dis 2011;17:2184-91.

15. PatientsLikeMe. 2005-2011. (http://www.patientslikeme.com/.) Accessed 14 November 2011.

16. Granovetter MS. The strength of weak ties. American Journal of Sociology 1973;78(6):1360-80.

17. Gladwell M. The Tipping Point. How Little Things Can Make a Big Difference. New York: Little, Brown and Company, 2000. 